\title{
ARACNÍDEOS PEÇONHENTOS: ANÁLISE DAS INFORMAÇÕES NOS LIVROS DIDÁTICOS DE CIÊNCIAS
}

\section{Venomous arachnids: an analysis of information in didactic science textbooks}

\author{
Adriano de Melo Ferreira ${ }^{1}$ \\ Cynthia Aparecida Arossa Alves Soares ${ }^{2}$
}

\begin{abstract}
Resumo: Um dos principais fatores responsáveis pela ocorrência de acidentes com aracnídeos peçonhentos é a falta de conhecimento da população sobre a biologia desses animais, bem como sobre a prevenção de acidentes. No caso do Ensino Fu ndamental, essas informações geralmente são abordadas nos livros de sexta série. Portanto, é muito importante que as informações contidas nos mesmos sejam suficientes e corretas, podendo contribuir para evitar que a integridade física do estudante seja prejudicada. Analisamos livros de Ciências aprovados pelo PNLD 2005 e verificamos que, mesmo após uma avaliação criteriosa por uma equipe de especialistas do MEC, estas obras ainda apresentam erros e insuficiências, exigindo dos professores uma análise mais crítica e detalhada das obras que serão escolhidas para suas aulas de Ciências.

Palavras-chave: Aracnídeos peçonhentos. Ensino de Ciências. Livros didáticos.

Abstract: One of the most important factors responsible for accidents that occur with venomous arachnids is the lack of information about the biology of these animals as well as accident prevention. Generally, this information is available in sixth year of basic education science's textbooks. Correct and sufficient information is very important for physical preservation of students. We analyzed science's textbooks approved by PNLD-2005 and verified that these books contain errors and insufficiencies, demanding a critical and detailed analysis by teachers.
\end{abstract}

Keywords: Venomous arachnids. Teaching of sciences. Didactic textbooks.

\footnotetext{
${ }^{1}$ Mestre em Biologia. Professor, curso de Licenciatura em Biologia, Universidade Estadual de Goiás (Unidade UnUcet) e Universidade Católica de Goiás (Departamento de Biologia). adrianodemeloferreira@bol.com.br> ${ }^{2}$ Mestre em Biologia. Professora, curso de Licenciatura em Biologia da Universidade Estadual de Goiás (Unidade UnUcet). <cyarossa@yahoo.com.br>
}

\footnotetext{
${ }^{1}$ Rua $229-A$, quadra 39-B Lote $1 / 28$ n 15 , esquina com rua 248

Setor Coimbra - Goiânia, GO

$74.535-240$ 
Ferreira, A. M.; Soares, C. A. A. A.

\section{Introdução}

De acordo com o Sistema Nacional de Informações Tóxico-Farmacológicas (SINITOX ), foram registrados no Brasil, no ano de 2006, 10.768 casos de intoxicação humana envolvendo aranhas e escorpiões (BRASIL, 2006a). Somente na região Centro-Oeste foram registrados 290 casos de acidentes com aranhas e 1.227 com escorpiões, perfazendo um total de 1.517 ocorrências (BRASIL, 2006b).

Além das condições precárias de habitação, outro principal fator responsável por esses índices é a falta de conhecimento da população geral sobre a biologia desses animais peçonhentos, bem como sobre a prevenção de acidentes. Informações estas que, geralmente, são abordadas durante as aulas das disciplinas "Ciências" (sexta série do Ensino Fundamental) e "Biologia" (segunda série do Ensino Médio). Porém, devido a alguns fatores, como a precariedade de recursos didáticos e a deficiência na formação dos professores, o mais utilizado dos recursos continua sendo o livro didático, o qual, "devido ao baixo poder aquisitivo da população e à elevada taxa de evasão escolar, talvez represente o único texto com que muitos brasileiros interagem durante suas vidas" (FRACALANZA, 1986, p. 28). Portanto, torna-se de suma importância que as informações contidas no mesmo sejam suficientes e corretas.

Preocupado com a qualidade de um recurso que é tão utilizado nas escolas brasileiras, em 1996, o Ministério da Educação e Cultura (MEC) implantou o Plano Nacional do Livro Didático (PNLD), que, por meio da ação de um grupo de especialistas, analisa e seleciona os livros ofertados pelo mercado editorial e, por intermédio de um guia distribuído nas escolas, permite aos professores escolherem apenas entre os livros aprovados. Bizzo (2000) considera importante esta iniciativa, pois as estratégias do mercado editorial são agressivas e a capacidade crítica do público-alvo é insuficiente. Nardi (1999) discorda desse autor, defendendo uma avaliação do livro didático pelos próprios professores, e não por uma equipe de especialistas.

Visando contribuir com a formação de docentes mais reflexivos e para um ensino de Ciências com qualidade nas escolas, este trabalho teve como objetivo realizar uma análise crítica das informações sobre aranhas e escorpiões de interesse médico nos livros didáticos de Ciências do Ensino Fundamental.

\section{A escolha e os critérios de análise dos livros}

Foram escolhidos para análise oito livros de Ciências da sexta série do Ensino Fundamental, que foram aprovados pelo PNLD de 2005 e constam do Guia de Livros Didáticos 2005 - quinta a oitava série. Tais obras (ALVARENGA et al., 2004; CRUZ, 2004; VALLE, 2004; BARROS e PAULINO, 2002; BORTOLOZZO e MALUHY, 2002; DE LA LUZ e SANTOS, 2002; GEWANDSZNAJDER, 2002; GOWDAK e MARTINS, 2002), foram analisadas quanto aos seguintes critérios: 1) o livro contém ilustrações (fotos ou desenhos) das principais espécies peçonhentas, facilitando a identificação pelo estudante? 2) o livro traz informações pertinentes aos principais aracnídeos de interesse médico, tais como: distribuição geográfica, habitat, tamanho, cor, comportamento (agressivo ou não) e sintomas em caso de acidentes? 3) apresenta medidas profiláticas? 4) o livro traz os procedimentos adequados a 
Aracnídeos peçonhentos: análise das informações...

serem adotados em caso de acidentes? 5) o livro aborda a importância ecológica desses animais?

\section{Resultados}

Os acidentes com animais peçonhentos constituem um problema relacionado não somente à saúde pública, mas também à economia de um país (BORGES, OLIVEIRA e COSTA, 2003). Um dos motivos para a ocorrência de acidentes é o desconhecimento, por parte da população, de algumas características dos animais causadores dos mesmos. Saber onde vivem, se possuem hábitos diurno ou noturno, se são agressivos ou não, entre outras informações, contribuiria para uma maior prevenção, sobretudo no caso de aracnídeos peçonhentos. E nesse contexto, o livro didático assume um papel muito importante, pois, como afirma Fracalanza (1986, p. 28), "este talvez represente o único texto com que muitos brasileiros interagem durante suas vidas".

No caso dos livros analisados, a maioria apresenta o tema "aracnídeos peçonhentos" no volume 6 (sexta série), dentro do capítulo sobre artrópodos, com exceção de Bortolozzo e Maluhy, que não abordam o assunto em nenhum dos volumes, e Gowdak e Martins (2002), que trazem o tema no volume 5 (quinta série). Alguns autores (BARROS e PAULINO, 2002; DE LA LUZ e SANTOS, 2002; GOWDAK e MARTINS, 2002) optaram por apresentar a temática dos acidentes em um quadro à parte do texto normal, que geralmente recebe nomes como: "Para ir mais além", "Fique informado", "Leitura" etc. Os demais apresentam as informações sobre aracnídeos causadores de acidentes na seqüência do texto normal, quando tratam de aracnídeos. E o número de páginas dedicadas ao assunto varia de um página e meia (ALVARENGA et al., 2004; BARROS e PAULINO, 2002; GEWANDSZNAJDER, 2002) a duas páginas e meia (VALLE, 2004).

Apenas um autor (CRUZ, 2004) aborda a distribuição geográfica, e, mesmo assim, de forma incipiente (Tabelas 1 e 2). A ausência dessa informação poderia dificultar o reconhecimento, pelo aluno, dos aracnídeos que comumente ocorrem na região em que vive. Por outro lado, a maioria das obras traz informações sobre o habitat, o que pode minimizar a deficiência sobre a distribuição geográfica. Quanto ao comportamento ser agressivo ou não uma informação de relevância no caso de aracnídeos peçonhentos -, é abordado pela metade dos autores, porém apenas no caso de aranhas (Tabela 1). Isso é compreensível visto que esse não é um critério muito considerado para escorpiões.

Santomé (1998) chama a atenção para o fato de a linguagem, terminologia e ilustrações dos livros-textos, muitas vezes, serem complexas e de difícil compreensão. Se disseminarem informações errôneas, então, agrava ainda mais a situação. Borges, Oliveira e Costa (2003), por exemplo, acreditam que um dos motivos para a propagação errônea das características apenas dos Viperídeos como serpentes peçonhentas seria a reprodução destas informações em livros didáticos. Três das obras analisadas (DE LA LUZ e SANTOS, 2004; GEWANDSZNADJER, 2002; GOWDAK e MARTINS, 2002) apresentam erros na ilustração de uma mesma aranha, a viúva-negra, apresentando a foto de outra aranha. Além disso, os autores ainda descrevem essa aranha com características de cor que nada têm a ver com a foto apresentada. Das outras cinco obras analisadas, somente três tiveram suas ilustrações consideradas 
Ferreira, A. M.; Soares, C. A. A. A.

satisfatórias, sobretudo a obra de Valle (2004), que traz as melhores ilustrações, com fotos grandes, bem nítidas e de fácil reconhecimento, apesar de, no caso dos escorpiões, apresentar fotos somente do escorpião-amarelo. Ainda em relação a informações errôneas ou insuficientes, sabe-se que todos os escorpiões de importância médica no Brasil pertencem ao gênero Tytius, representando $60 \%$ da fauna escorpiônica neotropical (LUCAS e SILVA-JÚNIOR, 1992). Apesar disso, alguns autores (DE LA LUZ e SANTOS, 2004; VALLE, 2004; GOWDAK e MARTINS, 2002; GEWANDSZNAJDER, 2002) se referem apenas a duas espécies de Tytius como sendo de interesse, enquanto outros (ALVARENGA et al., 2004; CRUZ, 2004) tratam de forma geral os escorpiões, como se todos fossem de importância médica (Tabela 2).

Incentivar a adoção de medidas profiláticas e divulgar os procedimentos corretos a serem adotados logo após um acidente, contribui para uma redução do número de casos com seqüelas, bem como representa uma economia para o serviço público de saúde. Nesse contexto, o livro didático, como recurso bastante utilizado nas salas de aula, não poderia jamais deixar de apresentar tais informações. Contudo, não foi o que se observou na análise realizada, pois a maioria das obras não apresenta medidas profiláticas, tanto para acidentes com aranhas como para os com escorpiões (Tabelas 1 e 2). No que tange aos procedimentos adotados em caso de acidentes, somente um autor (CRUZ, 2004) aborda noções de primeiros socorros, enquanto os demais autores apenas sugerem o encaminhamento da vítima para o hospital. Portanto, nesse aspecto, as obras analisadas deixam a desejar e merecem ser repensadas. Contudo, para evitar o risco de se prescreverem medidas preventivas e de tratamento erradas, os autores de livros didáticos deveriam consultar especialistas da área, evitando, assim, induzir comportamentos errôneos.

Outra informação pouco explorada em todos os livros analisados é a importância ecológica de aranhas e escorpiões, os quais somam, atualmente, mais de 35 mil espécies viventes (BRUSCA e BRUSCA, 2007) e têm um papel muito importante não só em relação aos acidentes que algumas poucas espécies causam, mas sobretudo para o meio ambiente. Nas obras analisadas, apenas Cruz (2004), Gewandsznajder (2002) e De la Luz e Santos (2002, sem referências para escorpiões) comentam a importância ecológica desses animais. Porém, vale ressaltar que mesmo esses autores fazem uma abordagem muito rápida, enfatizando somente a atuação desses animais como predadores de pragas. Como lembra Schwertner (2000, p. 27), infelizmente, "a abordagem dos seres vivos nas aulas de Ciências e Biologia, geralmente planejadas a partir dos livros didáticos, não escapa das visões que se faz da natureza, entre elas a antropocêntrica". Visão essa que, na maioria das vezes, traz representações de alguns animais como sendo feios, sujos, perigosos, nojentos, etc., contribuindo assim para o aumento da extinção desses animais. De acordo com Santos (2000), tais representações seriam reforçadas por filmes como Tubarão, Aracnofobia (o que dizer de um filme com o título "Aranhas malditas", por exemplo?), além das revistas de divulgação científica e livros didáticos. Esse mesmo autor questiona se não seria o momento de "promover" esses animais, destacando a importância ecológica dos mesmos. Sugere ainda que até mesmo a forma como nomeamos esses animais pode contribuir para a sua preservação. 
Aracnídeos peçonhentos: análise das informações...

Tabela 1. Ocorrência de informações importantes sobre aranhas peçonhentas nos principais livros didáticos de Ciências (6 ${ }^{\mathrm{a}}$ série do Ensino Fundamental) aprovados pelo PNLD 2005.

\begin{tabular}{|c|c|c|c|c|c|c|c|c|c|}
\hline \multicolumn{2}{|c|}{ Livros } & Ilustrações & \multicolumn{3}{|c|}{$\begin{array}{c}\text { Distribuição } \\
\text { geográfica }\end{array}$} & Habitat & \multicolumn{2}{|c|}{ Tamanho } & Cor \\
\hline \multicolumn{2}{|c|}{ 1. Cruz, D., 2004} & $X *$ & \multicolumn{3}{|c|}{$x *$} & $x$ * & \multicolumn{2}{|c|}{-- } & -- \\
\hline \multicolumn{2}{|c|}{ 2. Gewandsznajder, F., 2002} & $x *$ & \multicolumn{3}{|c|}{ - } & - & \multicolumn{2}{|c|}{$x *$} & $X *$ \\
\hline \multicolumn{2}{|c|}{ 3. Barros, C.; Paulino, W., 2002} & $x$ & \multicolumn{3}{|c|}{-- } & - & \multicolumn{2}{|c|}{$x$ * } & $x$ * \\
\hline \multicolumn{2}{|c|}{ 4. Alvarenga et al., 2004} & $x$ * & \multicolumn{3}{|c|}{-- } & $X *$ & \multicolumn{2}{|c|}{$x$} & $x$ \\
\hline \multicolumn{2}{|c|}{ 5. Valle, C., 2004} & $x$ & \multicolumn{3}{|c|}{-- } & $x$ & \multicolumn{2}{|c|}{$x$} & $x$ \\
\hline \multicolumn{2}{|c|}{ 6. Gowdak, D.; Martins, E., 2002} & $X^{*}$ & \multicolumn{3}{|c|}{-- } & $X$ & \multicolumn{2}{|c|}{$x$} & $x$ \\
\hline \multicolumn{2}{|c|}{ 7. Bortolozzo, S.; Maluhy, S., 2002} & \multirow{2}{*}{-} & \multicolumn{3}{|c|}{-- } & - & \multicolumn{2}{|c|}{-- } & - \\
\hline \multicolumn{2}{|c|}{ 8. De La Luz, M.; Santos, M. T., 2002} & & \multicolumn{3}{|c|}{-- } & $x$ & \multicolumn{2}{|c|}{$x$} & $x$ \\
\hline Livros & Comportamento & $\begin{array}{l}\text { Sintomas de } \\
\text { acidentes }\end{array}$ & $\begin{array}{l}\text { Medidas } \\
\text { profilátic }\end{array}$ & & $\begin{array}{l}\text { Proc } \\
\text { caso }\end{array}$ & $\begin{array}{l}\text { edimento } \\
s \text { de acid }\end{array}$ & em & $\begin{array}{r}\text { Impc } \\
\text { eco }\end{array}$ & $\begin{array}{l}\text { rtância } \\
\text { lógica }\end{array}$ \\
\hline 1. & -- & $X$ * & - & & & $x$ & & & $x$ \\
\hline 2. & - & $x$ & $x$ & & & $x$ * & & & $x$ \\
\hline 3. & - & $x$ & - & & & $X^{*}$ & & & -- \\
\hline 4. & $X *$ & $X *$ & - & & & $x$ * & & & -- \\
\hline 5. & $X^{*}$ & - & $x$ & & & $X *$ & & & -- \\
\hline 6. & $x$ & $X^{*}$ & - & & & - & & & -- \\
\hline 7. & - & -- & -- & & & - & & & -- \\
\hline 8. & $x$ & $x$ & - & & & -- & & & $X^{*}$ \\
\hline
\end{tabular}

$X$ = A obra apresenta informações satisfatórias quanto ao critério; $X^{\star}$ = Apresenta informações errôneas ou incipientes; -- = não apresenta a informação quanto ao critério

Tabela 2. Ocorrência de informações importantes sobre escorpiões peçonhentos nos principais livros didáticos de Ciências (6 ${ }^{\mathrm{a}}$ série do Ensino Fundamental) aprovados pelo PNLD 2005.

\begin{tabular}{|c|c|c|c|c|c|c|c|c|c|}
\hline \multicolumn{2}{|c|}{ Livros } & Ilustrações & \multicolumn{3}{|c|}{$\begin{array}{l}\text { Distribuição } \\
\text { geográfica }\end{array}$} & Habitat & \multicolumn{2}{|c|}{ Tamanho } & Cor \\
\hline \multicolumn{2}{|c|}{ 1. Cruz, D., 2004} & $x *$ & \multicolumn{3}{|c|}{$x^{*}$} & $X *$ & \multicolumn{2}{|c|}{-- } & -- \\
\hline \multicolumn{2}{|c|}{ 2. Gewandsznajder, F., 2002} & $x$ & \multicolumn{3}{|c|}{-- } & -- & \multicolumn{2}{|c|}{-- } & -- \\
\hline \multicolumn{2}{|c|}{ 3. Barros, C.; Paulino, W., 2002} & $x$ & \multicolumn{3}{|c|}{-- } & -- & \multicolumn{2}{|c|}{-- } & -- \\
\hline \multicolumn{2}{|c|}{ 4. Alvarenga et al., 2004} & $X$ * & \multicolumn{3}{|c|}{-- } & -- & \multicolumn{2}{|c|}{-} & -- \\
\hline \multicolumn{2}{|c|}{ 5. Valle, C., 2004} & $x$ & \multicolumn{3}{|c|}{-- } & $x$ & \multicolumn{2}{|c|}{$x$} & $x$ \\
\hline 6. Gowdak, & Martins, E., 2002 & -- & \multicolumn{3}{|c|}{-- } & $x$ & \multicolumn{2}{|c|}{ - } & -- \\
\hline \multirow{2}{*}{\multicolumn{2}{|c|}{$\begin{array}{l}\text { 7. Bortolozzo, S.; Maluhy, S., } 2002 \\
\text { 8. De La Luz, M.; Santos, M. T., } 2002\end{array}$}} & \multirow{2}{*}{-- } & \multicolumn{3}{|c|}{-- } & - & \multicolumn{2}{|c|}{-- } & -- \\
\hline & & & \multicolumn{3}{|c|}{-- } & -- & \multicolumn{2}{|c|}{-} & -- \\
\hline \multicolumn{2}{|l|}{ Livros } & $\begin{array}{l}\text { Sintomas de } \\
\text { acidentes }\end{array}$ & $\begin{array}{r}\text { Medida } \\
\text { profilátic }\end{array}$ & & $\begin{array}{l}\text { Proc } \\
\text { caso }\end{array}$ & $\begin{array}{l}\text { dimento } \\
\text { de acid }\end{array}$ & $\begin{array}{l}\text { em } \\
\text { ntes }\end{array}$ & $\begin{array}{r}\text { Impc } \\
\text { ecc }\end{array}$ & $\begin{array}{l}\text { rtância } \\
\text { lógica }\end{array}$ \\
\hline 1. & -- & -- & -- & & & $x$ & & & $x$ \\
\hline 2. & -- & $x$ & $x$ & & & $X^{*}$ & & & $X$ \\
\hline 3. & - & - & $x$ & & & $X^{*}$ & & & -- \\
\hline 4. & - & $X^{*}$ & - & & & $X *$ & & & -- \\
\hline 5. & - & - & $X$ & & & $X^{*}$ & & & -- \\
\hline 6. & - & -- & -- & & & -- & & & -- \\
\hline 7. & -- & -- & -- & & & - & & & -- \\
\hline 8. & -- & - & -- & & & -- & & & -- \\
\hline
\end{tabular}

$\mathrm{X}=\mathrm{A}$ obra apresenta informações satisfatórias quanto ao critério; $\mathrm{X}^{\star}=$ Apresenta informações errôneas ou incipientes; -- = não apresenta a informação quanto ao critério. 
Ferreira, A. M.; Soares, C. A. A. A.

Um aspecto observado bastante interessante é que, em relação a todos os critérios analisados, existem mais informações sobre aranhas que escorpiões nos livros didáticos que foram avaliados. Isso é um tanto incoerente já que, anualmente, são registrados mais casos de acidentes com escorpiões. Essa maior ênfase para as aranhas também poderia levar o estudante a pensar erroneamente que esses animais são mais importantes que os escorpiões. A obra que contemplou mais critérios suficientes sobre escorpiões foi a de Gewandsznajder (2002), enquanto o livro de De la Luz e Santos (2002) se destacou com informações sobre aranhas (Tabelas 1 e 2).

\section{Considerações finais}

Sabemos que a solução para dificuldades de aprendizagem e mudanças de conceitos prévios errados não depende apenas da qualidade dos livros didáticos adotados nas escolas. Esse é um processo muito mais complexo e depende de fatores que vão desde a formação dos docentes até a história pessoal de cada aluno, que interfere na sua maneira de interagir com o conhecimento adquirido na escola. Porém, a melhoria dos textos didáticos pode contribuir com sua parcela para a melhoria das condições de ensino/aprendizagem.

A análise realizada reflete bem o que ocorre com a maioria dos livros didáticos adotados no ensino de Ciências de sexta série, e nos permite concluir que, mesmo após uma avaliação criteriosa por uma equipe de especialistas do MEC, essas obras ainda apresentam erros e insuficiências em relação ao tema. Como principais conseqüências disso, podemos destacar a falta de orientação adequada aos estudantes para a prevenção de acidentes com aracnídeos peçonhentos, e o risco de extinção de animais temidos pela população, que não possui conhecimentos básicos sobre a importância ecológica desses animais.

Tais problemas exigem dos professores uma análise mais detalhada das obras antes da escolha das mesmas para adoção em suas aulas, não dependendo tão-somente da análise feita por profissionais que se encontram, geralmente, em uma esfera tão distante das realidades das salas de aula. Esperamos que nosso trabalho possa ajudar nessa tarefa já que, enquanto este artigo estava sendo preparado para publicação, o Fundo Nacional de Desenvolvimento da Educação (FNDE) divulgou a relação dos livros didáticos aprovados para constar no Guia de 2008 (BRASIL, 2007). A maioria das obras analisadas aqui consta desse novo guia. 
Aracnídeos peçonhentos: análise das informações...

\section{Referências}

ALVARENGA, J. P. et al. Ciências naturais no dia-a-dia, $\mathbf{6}^{\mathbf{a}}$ série: manual do professor. Curitiba: Nova Didática, 2004.

BARROS, C.; PAULINO, W. R. Ciências - ss seres vivos, $\mathbf{6}^{\mathbf{a}}$ série: manual do professor. São Paulo: Ática, 2002.

BIZZO, N. Falhas no ensino de Ciências. Revista Ciência Hoje, v. 27, n. 159, p. 26-31, 2000.

BORGES, R. C.; OLIVEIRA, A.; COSTA, R. M. C. Serpentes peçonhentas: identificação e procedimentos em acidentes - uma análise dos livros didáticos do Ensino Fundamental e Médio. Revista de Estudo e Pesquisa em Educação, v. 5, n. 1, p. 121-133, 2003.

BORTOLOZZO, S.; MALUHY, S. Link da Ciência, $\mathbf{6}^{\mathbf{a}}$ série: manual do professor. São Paulo: Moderna, 2002.

BRASIL. Ministério da Educação. Guia de livros didáticos PNLD 2008: Ciências. 106 p. Disponível em: <ftp://ftp.fnde.gov.br/web/livro_didatico/

guias_pnld_2008_ciencias.pdf>. Acesso em: 22 abr. 2007.

BRASIL. Ministério da Saúde. FIOCRUZ/SINITOX. Tabela 4 - Casos registrados de Intoxicação Humana, de Intoxicação Animal e de Solicitação de Informação por Agente Tóxico. Brasil, 2006a. Disponível em: <http://www.fiocruz.br/sinitox/2006/ tab4_brasil.pdf>. Acesso em: 26 ago. 2008.

Tabela 2 - Casos registrados de Intoxicação Humana, de Intoxicação Animal e de Solicitação de Informação por Agente Tóxico. Região Centro-Oeste, 2006b. Disponível em: <http://www.fiocruz.br/sinitox/2006/tab2_centro_oeste.pdf>. Acesso em: 26 ago. 2008.

BRUSCA, R. C.; BRUSCA, G. J. Invertebrados. 2. ed. Rio de Janeiro: Guanabara Koogan, 2007.

CRUZ, D. Ciência \& Educação Ambiental, $6^{a}$ série: manual do professor. São Paulo: Ática, 2004.

DE LA LUZ, M.; SANTOS, M. T. Coleção vivendo Ciências, $\mathbf{6}^{\mathbf{a}}$ série: manual do professor. São Paulo: Editora FTD, 2002.

FRACALANZA, H.; AMARAL, I. A.; GOUVEIA, M. S. F. O ensino de Ciências no $1^{\circ}$ grau. São Paulo: Atual Editora, 1986.

GEWANDSZNAJDER, F. Ciências e vida na Terra, $\mathbf{6}^{\mathbf{a}}$ série: manual do professor. São Paulo: Ática, 2002

GOWDAK, D.; MARTINS, E. Coleção Ciências, novo pensar, $5^{\mathbf{a}}$ série: manual do professor. São Paulo: Editora FTD, 2002. 
Ferreira, A. M.; Soares, C. A. A. A.

LUCAS, S. M.; SILVA JR, P. I. Acidente por escorpiões I: Escorpiões de Interesse Médico no Brasil. In: Schvartsman, S. (Ed.). Plantas Venenosas e Animais Peçonhentos. São Paulo: Sarvier, 1992. p. 211-215.

NARDI, R. A avaliação de livros e materiais didáticos para o ensino de Ciências e as necessidades formativas do docente. In: BICUDO, M. A. V.; JUNIOR, C. A. S. (Orgs.). Formação do educador e avaliação educacional. São Paulo: Editora Unesp, 1999. v. 4. p. 93-103.

SANTOMÉ, J. T. Globalização e interdisciplinariedade: o currículo integrado. Porto Alegre: Artes Médicas, 1998.

SANTOS, L. H. S. Tem alguma utilidade estudar a utilidade dos seres vivos? In: (Org.). Biologia dentro e fora da escola: caderno de educação básica. 6. ed. Porto Alegre: Editora Mediação, 2000. p. 13-24.

SCHWERTNER, C. F. Os bichos na natureza da sala de aula. In: SANTOS, L. H. S. (Org.). Biologia dentro e fora da escola: caderno de educação básica 6. ed. Porto Alegre: Editora Mediação, 2000. p. 25-40.

VALLE, C. Vida e ambiente, $\mathbf{6}^{\mathbf{a}}$ série: manual do professor. Curitiba: Nova Didática, 2004.

Artigo recebido em abril de 2007 e aprovado em junho de 2008. 